\title{
Research on Estimation of Paddy Field Area Index Based on UAV Remote Sensing Images
}

\author{
Xiuli Lu and Yongli Yang \\ Baoding University of Technology Baoding, Hebei 071000 \\ E-mail: lixiuli342@gmail.com, Yangyongli86@outlook.com \\ Zhou Yang \\ Baoding Jing Xin Electric Co. LTD Baoding, Hebei 071000 \\ E-mail: zhouy7069@gmail.com \\ Amit Sharma \\ Department of Computer Science and Engineering, Chitkara University, Punjab, India \\ E-mail: amit.amitsharma90@gmail.com
}

Keywords: UAV image; rice; object-oriented segmentation; nearest neighbor supervised classification; planting area

Received: May 21, 2021

This paper takes the rice plot as the research object, and uses the portable UAV Mavic Pro for aerial photography. Preprocess the acquired UAV images to generate orthophotos with a resolution of $3.95 \mathrm{~cm} /$ pix. Using object-oriented thinking, visual evaluation and ESP tools are combined to quickly select the optimal segmentation scale to be 300, and support is applied. Vector machine, random forest, and nearest neighbor supervised classification methods have carried out ground object classification and rapid extraction of rice area. The classification results and area accuracy are evaluated by visual classification results. The method with the highest overall accuracy is the nearest neighbor classification method. At this time, the user accuracy of rice classification is $95 \%$, and the area consistency accuracy is 99\%. The results show that UAV remote sensing and automatic classification can quickly obtain high resolution images and extract rice planting area in plain rice planting area, make up for the lack of ground survey data when Nongshan is blocked, and provide samples and verification basis for the calculation of large-scale rice planting area, yield and other information.

Povzetek: Predstavljen je sistem za analizo UAV posnetkov za iskanje površin riža.

\section{Introduction}

Aerospace remote sensing monitors a very large scale, but it contains less information due to its low spatial resolution. On the contrary, terrestrial remote sensing has high spatial resolution to obtain rich information, but the monitoring range is limited. With the development of flight technology. UAV platforms with flexible maneuverability, strong operation selectivity, high accuracy, short operation cycle, good timeliness, low maintenance and usage costs, economical and practical, and good safety have matured in recent year [1].

With the rapid development of microcomputers, communication equipment and other technologies, the technology of drones equipped with remote sensing has the characteristics of flexible flight, wide monitoring range, and rich data acquisition [2]. The image stitching method is selected to obtain highresolution pictures. Interpret the picture with color feature, texture feature and shape feature, select the appropriate classification method, construct the accurate extraction and measurement of crop area, provide a new method for rapid extraction of planting

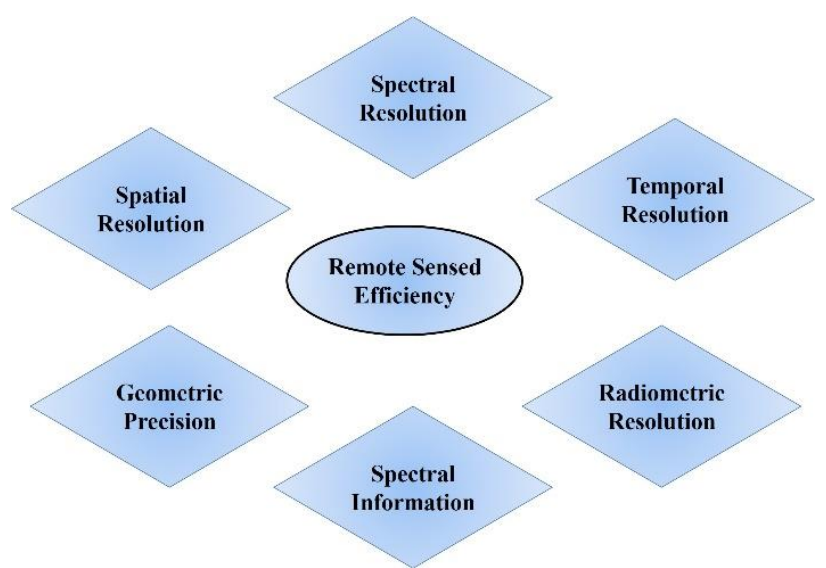

Figure 1: UAV's efficiency for precision agriculture.

area, and meet the strategic requirements of the national precision agriculture development [3].

Figure 1, depicts some of the concerns which influences the superiority of remote sensed images and are highlighted in this article. Remote sensing-based images for agricultural application can be implemented for planning soil assessments, crop species classification, water stress detection for crop yield, 
monitoring and detecting diseases and weeds of crops, and representing of crop yield. The importance of remote sensing in precision agriculture through various platforms such as UAV's, Satellite or ground deployed sensors has gained attention. These various platforms provide real time collection of data, spectral bands in width and number collected through sensors, spatial resolution information for high, low and medium values, temporal resolution based on hourly, daily, weekly information, radiometric information about 8bit, 12-bit and 16-bit resolutions are collected and analyzed in ground station. These collected information in real time are analyzed in ground station for decision making and many of the concerns are addressed which includes, the accuracy of matching the collected images with exact ground location. The other addressed concerns are evaluating the extent of spatial resolution and spectral resolution features extracted from images and also analyzing the quality of information which is acquired from the images. Some studies use UAV's for the collection of data and its analysis in real time through remote station for the application of forest fire detection $[4,5]$.

One of the most essential grained crops across the word is Rice, specifically in Asian countries. China is one of the leading countries in Asia for rice graining. In China more than $60 \%$ of population grain rice as their staple food [6]. The correct knowledge of price building is essential for ensuring the security of food and promoting the overall development. Remote sensing is a procedure through which one can obtain the required information about anything through sitting at remote places. The remote sensing provides the capability for obtaining spectra data which transmits the essential information and indicates the interaction among solar radiation like scattering and absorption of vegetation.

The rest of the article is organized as the most recent work done in the field of agricultural monitoring is highlighted in Section 2. The process of data acquisition and pre-processing is described in Section 3. Image segmentation and classification methods are described in Section 4 that is followed by the experimental evaluation and its analysis in Section 5. The discussions drawn from the experiment is described in Section 6 which is followed by the conclusion in Section 7.

\section{Literature review}

In order to eliminate the influence of spectral mixing on crop mapping accuracy, Liu et al. proposed an innovative method to generate field canopy height data by calculating the elevation difference between vegetation and non-vegetation plots. The support vector machine is applied to four types of data sets: 1 ) pixel-based spectral features (PSF); 2) PSF and canopy height features; 3) object-based spectral features (OSF); and 4) object-based Spectral characteristics and canopy height characteristics (OSCHF). The results show that OSCHF has the highest classification accuracy rate for all categories, with an overall accuracy of $94.04 \%$, Kappa of 0.91 , which is significantly higher than the result of OSF, and the worst accuracy of PSF. OSF can eliminate the speckle noise problem to some extent, but due to the similarity of spectra, grapes and trees are still misclassified as rice to some extent. Fortunately, these confusions can be effectively avoided by including the height of the canopy [7]. Dai et al. selected the Gemini MyFlyDream MTD fixed-wing drone equipped with Canon EF-M 18-55 cameras to obtain visible light images of the 135th Regiment of the Eighth Division of Xinjiang Construction Corps. In-house cotton planting information was extracted. The cotton planting area extracted by visual interpretation was $0.35 \mathrm{~km} 2$, the cotton planting area extracted by objectoriented extraction was $0.33 \mathrm{~km} 2$, the classification result accuracy was $94.29 \%$, and the error coefficient was $5.71 \%$, which can be effectively extracted and studied. Regional cotton planting information. Compared with the traditional pixel-based classification method, the object-oriented classification method has higher extraction accuracy and is closer to the extraction result of visual interpretation [8]. Zhang et al. used UAV low-altitude remote sensing data as samples and GF-2 remote sensing images as data sources, combined with field surveys, to extract the planting area of Salvia miltiorrhiza in Luoning County. Standard processing remote sensing satellite data can obtain specific remote sensing data coverage. Preprocess the drone data to intuitively explain the types and distribution of Chinese medicine resources in the samples. The support vector machine (SVM) was used to classify and estimate the traditional Chinese medicine resources in Luoning County, and the confusion matrix was used to determine the accuracy of the spatial distribution of traditional Chinese medicine resources. The results show that the application of low-altitude remote sensing technology for unmanned aerial vehicles and satellite remote sensing images is feasible in the extraction of South African ginseng and other varieties of planting areas, and it also provides a scientific reference for the poverty alleviation policy of traditional Chinese medicine. At the same time, actively carry out the remote sensing classification research of Chinese medicinal materials based on multi-source and multiphase high-resolution remote sensing images to explore more effective methods of extracting Chinese medicinal materials [9].

In recent years there have been many researchers reported in the application of precision agriculture, such as detection of diseases in crop, estimation of crop yields, fruit grading, detection of weeds and many others [10]. For a specific issue, various researchers have designed specific methods for resolving these issues. The most appropriate approach for smart agriculture is the combination of machine learning and image pre-processing methods [11]. The image preprocessing-based approaches consist various stages such as contrast stretching, mask processing, point 
processing, histogram equalization and many other processing approaches. While on the other hand machine learning methods consists of various approaches such as neural networks [12], fuzzy logic [13], K-nearest neighbors [14], self-adaptive maps, support vector machines [15] and many others. Considering all the approaches of machine learning, the artificial neural networks has gained attention from various researchers across the globe [16].

The spectra for the vegetation is equivalent to the vegetation growth. The vegetation supports absorption of light near visible range due to the presence of less reflectance. On the other hand the vegetation reflectance near infrared range is comparatively which gets easily affected by tissues and structure of thick plants. There exist many studies which has been developed in relation to the vegetation spectra considering the vegetation growth limitations such as chlorophyll molecules, leaf area index, biomass and therefore majority of vegetation indices are computed through the reflectance of various ranges of spectra. Many studies have been proposed for accurately estimating these parameters, such as Candiago et al. [17] have utilized enhanced vegetation index along with wide dynamic range vegetation index which has been obtained through MODIS for the accurate evaluation of crop productivity with observed variation coefficient for maize below $20 \%$ and for soybean below $25 \%$.

The authors in [18] have implemented various spectral indices on the basis of red edge fore estimating the nitrogen uptake of plant with determination coefficient about $76 \%$. The parametric statistical method on the basis of vegetation indices is considered as the simplest and most efficient among estimation approaches weekend be used for monitoring the growth [19]. The changing status of crop growth candy efficiently monitor through spectral measures that directly calculates its yield percentage. Therefore, the vegetation indices considered as most valuable for estimating the crop yield remotely over large scale [20]. The authors in [21] happy propose system for estimating the wheat yield capacity in Ukraine and Kansas. Present $7 \%$ of error by implementing time series normalized difference vegetation index which is obtained from MODIS. The authors in [22] presented a new approach for estimating the crop yield through to the temperature vegetation dryness index by computing the RMSE coefficients in range between $10 \%$ to $14 \%$ for soybean and $15 \%$ to $23 \%$ for wheat.

Sharma et al. have described the block chain technology for e-healthcare services and industrial application [23, 24]. The authors in [25] proposed an approach for successfully mapping the crop yields by implementing wide dynamic range vegetation index which is derived from the MODIS time series data and the computed estimation error is observed as below $30 \%$ at state level. The vegetation indices-based approaches the most efficient approach for the prediction of crop yield and multiple regression algorithms for utilizing the vegetation indices are implemented for estimating the crop yield. The regression method-based estimation of crop yield which includes simple linear and complex nonlinear functions [26]. It is observed from many of experiments that the utilization of accurate vegetation indices is an essential parameter for estimating the crop yield accurately instead of estimation of complex function structure. Therefore, it is considered as essential parameter for the evaluation of crop yield to get the accurate vegetation index. There may be a situation when a sensor node fails in a network, localization of unknown sensor node is essential for the network to work efficiently [27, 28]. The UAV systems has a great potential in the application of agricultural monitoring and also addresses various constraints in other research areas. It provides the facility of remote sensing through which an agricultural field can be monitored remotely without any transportation required [29]. The UAV system operates as a standalone device which are limited to a group of UAVs incorporating a stable architecture means that the system is able to work properly, even when there is no inter communication among UAVs [30]. The idea behind this research is to collect field data through UAVs equipped with IR sensors and capture images in real time. The UAV transmits information to ground station for accessing the data in real time and make decisions. The prime objective of this research is to design UAV based system for implementing a UAV based design for monitoring agricultural fields in real time which includes framework, architecture and its components.

\section{Data acquisition and preprocessing}

Figure 2, presents the complete workflow of proposed remote sensing based architecture of paddy crop field monitoring system. It mainly consists of five operational stages.

In first stage real time image are captured from the UAVs and transferred to the ground station. The second stage and third consists of pre-processing and sampling of collected images in ground station. The monitoring station applies various classifiers for the training and testing the features in order to predict the crop yield in fourth stage. In final stage the crop yield is prediction based on the classifier and final decisions and outcomes are suggested to the workers and farmers. 


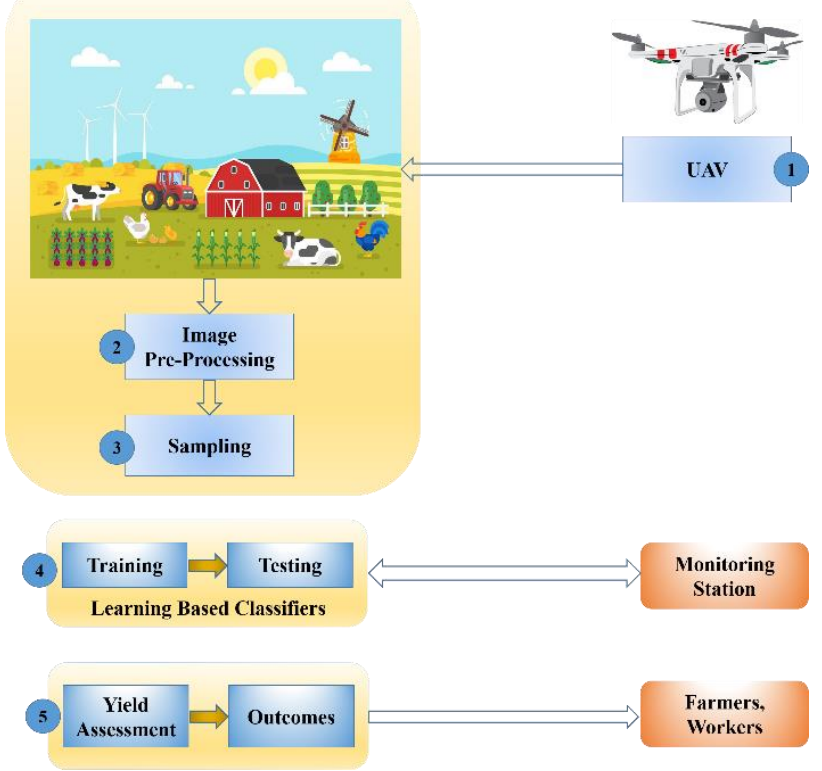

Figure 2: Workflow of field assessment based on UAVs and classifier techniques.

Multispectral and digital images were collected from the IR cameras which are installed in UAV. The first stage is pre-processing where collected images are processed and analyzed which is followed by organization of the several images, using Agisoft PhotoScan software. The collected images were analyzed based on their region of interest and red, green and blue components are extracted. On the basis of RGB values the color index is calculated and further processed utilizing digital number. The values of digital number for RGB channels reflects the performance of reflectance and radiance in visible range of crop canopy. The normalized values of digital number for RGB are computed which is followed by the vegetation approximation in comparison with original digital number values. The transformation process of raw pixel indices into reflectance is further analyzed. The converted raw pixels from image are converted to radiance units as per the specified process of the calibration model. The average radiance value for pixels that are observed inside original panel of image are computed.

\subsection{Data acquisition}

Civilian UAVs can be divided into four categories: unmanned fixed-wing aircraft, rotary-wing UAVs, para-wing UAVs and other types of autonomous flying equipment. The DJ-Innovations design and production of UAVs with Mavic Pro models in this study is shown in Table 1. The main parameters are shown in Table 1. The integrated design of the drone's three-axis stabilization gimbal and visible light camera ensures the quality of the captured images [31, 32]. Mavic Pro adopts a foldable design and is equipped with 4 foldable cantilever propellers. It takes less than 1 minute from deployment to take-off. With DJI GS Pro software, it can realize autonomous route planning and surveying in the sample plot, which can meet the needs of field agricultural sampling process. The portability of the equipment and the quickness of use are required to improve the sampling efficiency.

The data of this study was obtained by aerial photography of the portable UAV Mavic Pro on the experimental plot $(113.780 \mathrm{E}, 30.760 \mathrm{~N})$ in a certain village on May 29, 2017. The experimental plot is mainly rice planting, and many crops such as lotus root and vegetables are also planted. The plot and the road are blocked by houses and trees, and the effective pictures collected by the original GVG system cannot be used. The weather was fine during takeoff, and the wind speed on the ground was less than level 4, suitable for aerial photography. Set the drone aerial photography height to $200 \mathrm{~m}$, camera shutter speed $1 / 25 \mathrm{~s}$, sensitivity 100 , heading overlap $70 \%$, shooting area area is about $26000 \mathrm{~m} 2$, and the spatial resolution can reach $0.04 \mathrm{~m}$. Through real-time viewing of the images, it was confirmed that images containing rice, lotus root, vegetables and other crops were obtained.

\subsection{Data pre-processing}

The drone images are spliced by Agisoft Photo Scan software, the entire workflow can be set to be automatically completed by the software, and the GPU can be used to accelerate the processing process. When aligning the imported images, the software will automatically arrange the images according to the image coordinates, elevation information, and similarity and find points with the same name [33]. The software is based on the estimated camera position. The program corresponding to each camera will calculate the depth information and combine it into a dense point cloud. After the dense point cloud is generated, a 3D model of the polygon mesh is generated based on the point cloud. Finally, a highresolution orthophoto (DOM) with real coordinates is generated, and the resolution of the generated orthoimage is $3.95 \mathrm{~cm} /$ pix and the projection type is WGS 84/UTM zone 49N (EPSG: 32649). The images are stitched globally and stored in a TIFF file.

\section{Object-oriented image segmentation and classification methods}

UAV images have the characteristics of extremely high spatial resolution and bright colors, which can express the texture, shape, topological relationship and other aspects of ground objects in more detail. Objectoriented classification methods are widely used in high-resolution image classification, so they are also suitable for UAV image segmentation and classification. In the object-oriented classification process, the basic processing unit is changed from a single pixel to an image object composed of multiple pixels of the same nature, so that the signal-to-noise ratio is significantly increased, and the "salt and pepper" phenomenon of traditional pixel-oriented 


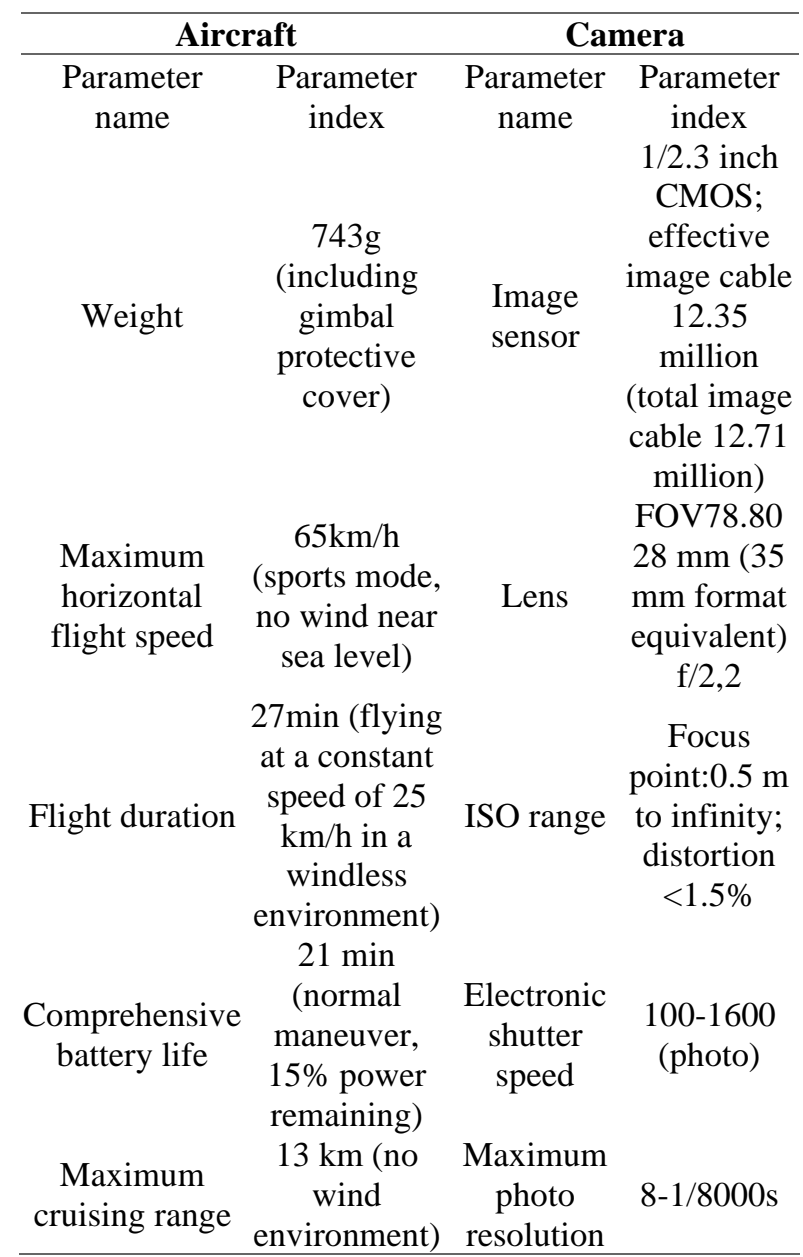

Table 1: Mavic Pro UAV main parameters.

processing results is eliminated up [34-36]. In objectoriented classification, in addition to using the spectral information of the ground features, it also makes full use of the geometric information and texture information of the ground features. The classification basis is more diversified and the recognition ability of the ground feature categories is enhanced, so that the classification accuracy can be significantly improved.

\subsection{Object-oriented image segmentation}

The extraction of crop planting information includes crop classification and area statistics. The development of remote sensing image classification has changed from traditional manual discrimination to computer recognition processing. Manual classification is to obtain data through comprehensive ground observation, compare it with remote sensing image photos, and interpret and extract remote sensing image information. The efficiency and flexibility are poor, and the subjectivity is strong. The error is large, and the crop information obtained has great limitations. The computer recognition and classification is based on the extraction of remote sensing image feature information, and the remote sensing image classification is carried out through a variety of mathematical operations combined with production algorithms. It has high efficiency, fast speed, low

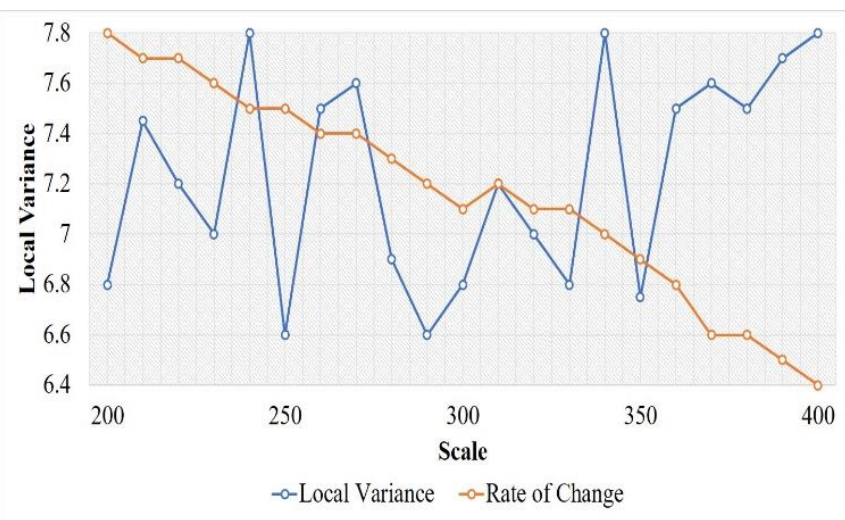

Figure 3: The optimal segmentation scale calculated by the ESP tool.

subjectivity and high accuracy. There are different methods for distinguishing different ground object categories for the acquired remote sensing images. At present, they are roughly divided into two categories: Based on pixel classification, this type of classification method only uses the spectral information of remote sensing images. The current development direction is multispectral development. Use remote sensing equipment to obtain as much spectral information as possible (such as near-infrared) through technical means, and classify the light reflectivity of different crops. The object-oriented law is to divide the image into individual objects through computer digital image processing technology and image classification algorithm technology, and classify them according to the similarity of object characteristics (spectrum, space, texture characteristics). The two classification methods are suitable for different remote sensing images, and the resulting images are also different.

The JPEG image used in the study only has the gray information of red, green and blue, and the spatial resolution is high. The spectral information based on a single pixel is used to separate the ground objects, and the shape and texture characteristics of the image are not classified in terms of classification. Utilization can easily lead to low classification accuracy, which will adversely affect the application of remote sensing images. Through the object-oriented classification method, instead of relying only on the spectral information of a single pixel, the object expands the set of image object units, and makes full use of attribute information such as spectral information, geometric features, texture features, and context. The accuracy of area extraction is more favorable. Therefore, the research first performs object segmentation on JPEG images, and then extracts multiple spatial feature values for the image object segmentation unit for processing analysis and statistics, and establishes a multi-feature object classification system. The data processing software uses eCognition Developer 9, the segmentation algorithm uses image multi-scale segmentation, and the classification algorithm uses specified class classification.

This paper uses 10 as the segmentation scale step size, and evaluates the segmentation effect by 
calculating the local variance of the homogeneity of image objects at different scales within 200400, and uses the ROC-LV (Rates of change of LV) value as the object segmentation maximum. Optimal scale parameters. As shown in Figure 3, the abscissa is the segmentation scale, and the ordinate is the local variance and the rate of change. When the segmentation scale is 300, the ROC-LV has a peak and maximum value. It is finally determined that the segmentation scale is 300 and the compactness is set to 0.5 , the shape factor is set to 0.1 , and the experimental image data is segmented. After segmentation, a total of 140 objects are generated, the smallest area is $2.39 \mathrm{~m} 2$, the largest area is $3162.79 \mathrm{~m} 2$, and the average area of each object is $225.61 \mathrm{~m} 2$.

\subsection{Image supervision classification}

Object-oriented classification can comprehensively use the distinctive spectrum, shape and texture of the UAV image segmentation object to classify images. Its basic classification methods are divided into two types: rulebased classification and supervised classification. The rule-based classification method is simple, and it can achieve better results for some features with obvious features, but it is poor for some features with similar features. However, the natural surface spectrum features are complex, there is the same spectral foreign body and the same object spectrum, there is no rule (set) can adapt to all UAV images. The need to make appropriate rules and select thresholds can be multiple times according to the image used. Commonly used supervised classification are nearest neighbor algorithm, support vector machine and random forest method. The supervised classification method mainly depends on the discriminant function to judge the characteristics of unknown things and the characteristics of limited samples to determine the category of samples to be divided. The regular classification method can also be applied to images with overlapping or overlapping features between classes. Therefore, this paper chooses to use supervised classification to classify the images in the experimental area.

After completing the segmentation of the sample plot images, visual interpretation is completed, and a system of 7 features including rice, trees, lotus root, vegetable field, bare land, roads and ditches is established. Configure the nearest neighbor feature space for the objects generated by segmentation, and select 24 samples for training, accounting for $17 \%$ of the segmented objects. The number of samples of rice, trees, lotus roots, vegetable plots, bare land, roads and ditches were $8,3,2,2,4,3$, and 2 , respectively. Because the classification accuracy is not directly proportional to the number of classification features, redundant classification features will cause an increase in calculation, a decrease in classification efficiency, and even a decrease in classification accuracy, so based on the characteristics of each category sample, compare 15 features in the RGB spectrum, shape, texture of each object of the selected class as the initial feature set. Figure 4, depicts the relationship between the separability (distinguishment distance) between classes and the number of features used for classification. Use the Feature Space Optimization tool in eCognition software to find the feature combination that can produce the maximum average minimum distance between different types of samples.

It can be seen that as the feature dimension increases, the change in the discrimination distance between samples at the beginning increases significantly, but the discrimination distance changes less after the feature dimension is greater than 6 , and the discrimination distance even decreases when the feature dimension is greater than 10 . The phenomenon. Comprehensive consideration of calculation amount and classification accuracy, and at the same time, determine the selection of 6 feature combinations of red spectrum average, yellow spectrum standard deviation, blue spectrum standard deviation, maximum difference measure, shape density, and gray level cooccurrence matrix contrast as the optimal feature according to the optimization results. Space, so as to avoid the problems such as the rapid increase of calculation, the decrease of classification accuracy, and the redundancy of classification features caused by the blind use of multiple features in the classification process.

After selecting the optimal feature space and class of interest samples, the nearest neighbor classification, support vector machine and random forest method are used to classify the image objects.

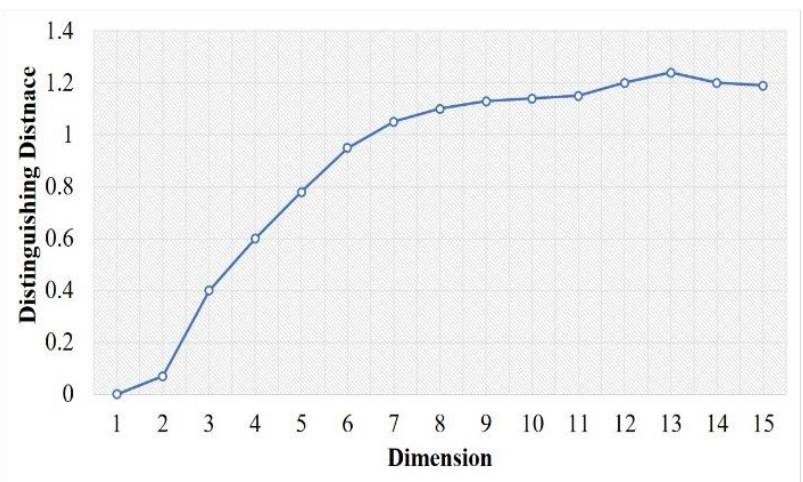

Figure 4: Relationship between the separability (distinguishment distance) between classes and the number of features used for classification.

\section{Evaluation and analysis}

In order to verify the superiority and effectiveness of extracting rice information based on UAV images, two methods are used to analyze the results, one is to use the calculation confusion matrix to evaluate the classification accuracy, and the other is to compare the extracted rice area with Compare the actual rice area and evaluate the area. Considering the high spatial resolution of UAV images, the verification data can be obtained through manual visual interpretation. 


\begin{tabular}{|c|c|c|c|c|c|c|c|c|c|}
\hline Classification & Feature category & Rice & $\begin{array}{c}\text { Lotus } \\
\text { root }\end{array}$ & Trees & $\begin{array}{l}\text { Bare } \\
\text { land }\end{array}$ & $\begin{array}{c}\text { Vegetable } \\
\text { field }\end{array}$ & The way & Ditch & $\begin{array}{c}\text { User } \\
\text { accuracy \% }\end{array}$ \\
\hline \multirow{10}{*}{$\begin{array}{c}\text { Nearest } \\
\text { neighbor } \\
\text { method }\end{array}$} & Rice & 42 & & & & 1 & 1 & & 95 \\
\hline & Lotus root & & 2 & 1 & & & & & 67 \\
\hline & Trees & & & 9 & & 2 & 1 & & 75 \\
\hline & Bare land & & & & 12 & 1 & & & 92 \\
\hline & Vegetable field & & & & & 6 & & & 100 \\
\hline & the way & & & & 1 & 1 & 5 & & 71 \\
\hline & ditch & & & & & & 1 & 3 & 75 \\
\hline & Drawing accuracy $/ \%$ & 100 & 100 & 90 & 92 & 55 & 83 & 60 & \\
\hline & Overall accuracy $/ \%$ & 89 & & & & & & & \\
\hline & Kappa coefficient & 0.84 & & & & & & & \\
\hline \multirow{10}{*}{$\begin{array}{c}\text { Support } \\
\text { vector } \\
\text { machine }\end{array}$} & Rice & 42 & & & & & & & 100 \\
\hline & Lotus root & & 2 & 1 & & 2 & & & 40 \\
\hline & Trees & & & 9 & & 2 & & & 100 \\
\hline & Bare land & & & & 12 & 1 & & & 88 \\
\hline & Vegetable field & & & & & 6 & & & 43 \\
\hline & the way & & & & 1 & 1 & 5 & & 100 \\
\hline & ditch & & & & & & 1 & 3 & 42 \\
\hline & Drawing accuracy $/ \%$ & 100 & 100 & 90 & 54 & 55 & 67 & 60 & \\
\hline & Overall accuracy $/ \%$ & 82 & & & & & & & \\
\hline & Kappa coefficient & 0.75 & & & & & & & \\
\hline \multirow{10}{*}{$\begin{array}{c}\text { Random } \\
\text { forest method }\end{array}$} & Rice & 42 & & 1 & & & & & 98 \\
\hline & Lotus root & & 2 & & & 1 & & & 67 \\
\hline & Trees & & & 8 & & & & 1 & 100 \\
\hline & Bare land & & & & 12 & 3 & & & 80 \\
\hline & Vegetable field & & & & & 7 & & 2 & 78 \\
\hline & the way & & & & 1 & & 4 & & 80 \\
\hline & ditch & & & & & & 2 & 3 & 50 \\
\hline & Drawing accuracy $/ \%$ & 100 & 100 & 80 & 92 & 64 & 67 & 60 & \\
\hline & Overall accuracy $/ \%$ & 88 & & & & & & & \\
\hline & Kappa coefficient & 0.73 & & & & & & & \\
\hline
\end{tabular}

Table 2: Confusion matrix of verification sample ground feature classification.

\subsection{Accuracy evaluation of classification results}

The accuracy evaluation of remote sensing image information classification results can be comprehensively evaluated through overall accuracy, mapping accuracy, user accuracy and kappa coefficient. For the images of the experimental area, 89 verification objects were randomly obtained through manual visual interpretation, and the verification points and the extraction results were compared to judge whether the extracted ground object classification results were correct. After statistical calculations, the method for verifying the overall accuracy of UAV images on the sample site is $89 \%$ of the nearest neighbor classification method, and the kappa coefficient is 0.84 . At this time, the mapping accuracy of rice is $100 \%$ and the user accuracy is $95 \%$. The specific results are shown in Table 2.
Although the images collected by drones only have three kinds of spectral information of red, green and blue, they have clearer shape and texture information than satellite remote sensing images. The spectrum, texture, and shape information of rice is more obvious and easy to distinguish compared to other ground objects. The average value of the red spectrum is to distinguish the color information of vegetation coverage objects (rice, trees, vegetable fields, lotus root) and non-vegetation coverage objects (bare land, water bodies, roads). The boundaries of trees are relatively broken, and the shape density is small.

The shape density can effectively distinguish rice and trees with close color averages. The texture feature gray-level co-occurrence matrix reflects the gray level of the ground object image with respect to direction, adjacent interval. The comprehensive information of the variation range, rice, trees, vegetable plots, lotus roots, bare land, water bodies and roads all have 
certain differences. It is the supplementary attribute to distinguish rice plots from the ground, so the rice classification accuracy is the highest. Roads, ditches, and other ground objects have a lot of intersections, and the classification accuracy is low. Some vegetable fields have similar characteristics with trees, and some have similar characteristics with bare land. Therefore, there will be mistakes in trees or bare land, and the classification accuracy is the lowest. But there are few cases of misclassification of rice, and the area of vegetable fields is generally not large, which will not have a big impact on the area of rice.

\subsection{Accuracy evaluation of area results}

Save the UAV image classification result as a shp file, use the geometric calculation function in ArcGIS to calculate the area of each object, and then perform summary statistics on each category to get the area of each feature type. The area consistency test method is as follows: the area of the visual interpretation result of different types of ground objects is the measured area $\mathrm{S} 1$, and the object-oriented classification result is the predicted area $\mathrm{S} 2$, then the relative area error e is:

$$
e=\frac{\left|S_{1}-S_{2}\right|}{S_{1}} \times 100 \%
$$

It can be seen from Table 3 that when the UAV image is used to extract the area of the ground feature, the UAV image has high resolution, bright color, clear ground feature shape and texture characteristics, and the UAV image and object-oriented classification method are applied to rice planting. The accuracy in area monitoring can be guaranteed, especially when the nearest neighbor method is used, the relative error is only $0.75 \%$

$$
\text { Yield }=\frac{1}{N} \sum_{e=1}^{N} Y_{e}
$$

The segmented images are labeled, and each yield value from the segmented images are further computed and the average yield value is used to produce the overall yield value of paddy fields by implementing Equation 2. Here $\mathrm{N}$ represents the amount of segmented images and $Y_{e}$ represents the yield

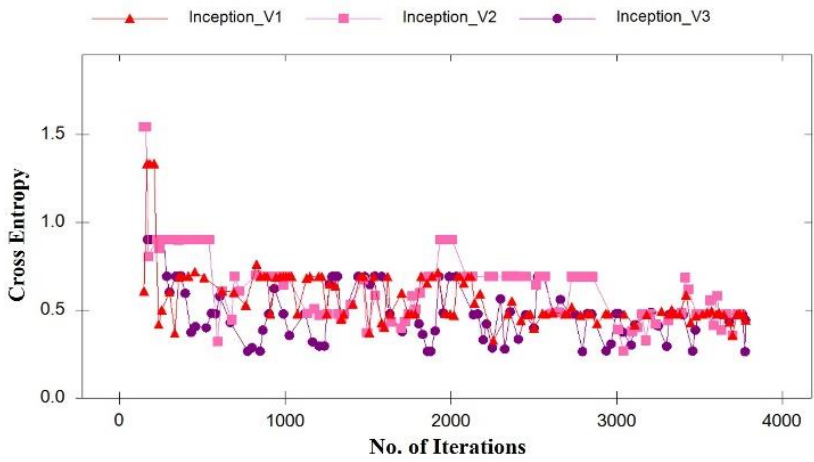

Figure 5: Cross entropy of three variants of

\begin{tabular}{|c|c|c|c|c|c|c|c|}
\hline \multirow[b]{2}{*}{$\begin{array}{l}\text { Cate- } \\
\text { gory }\end{array}$} & \multirow{2}{*}{$\begin{array}{c}\text { Measu- } \\
\text { red } \\
\text { area } / \mathbf{m}^{2}\end{array}$} & \multicolumn{2}{|c|}{$\begin{array}{c}\text { Nearest } \\
\text { neighbor } \\
\text { method }\end{array}$} & \multicolumn{2}{|c|}{$\begin{array}{l}\text { Support } \\
\text { vector } \\
\text { machine }\end{array}$} & \multicolumn{2}{|c|}{$\begin{array}{c}\text { Random } \\
\text { forest method }\end{array}$} \\
\hline & & $\begin{array}{c}\text { Fore- } \\
\text { cast } \\
\text { area/ } \\
\mathrm{m}^{2}\end{array}$ & $\begin{array}{l}\text { Rela- } \\
\text { tive } \\
\text { error/ } \\
\%\end{array}$ & $\begin{array}{c}\text { Fore- } \\
\text { cast } \\
\text { area/ } \\
\mathrm{m}^{2}\end{array}$ & $\begin{array}{c}\text { Rela- } \\
\text { tive } \\
\text { error/ } \\
\%\end{array}$ & $\begin{array}{c}\text { Fore- } \\
\text { cast } \\
\text { area/ } \\
\mathrm{m}^{2}\end{array}$ & $\begin{array}{l}\text { Rela- } \\
\text { tive } \\
\text { error/ } \\
\%\end{array}$ \\
\hline Rice & 16508 & 16385 & 0.75 & 15876 & 3.83 & 16112 & 2.40 \\
\hline $\begin{array}{l}\text { Bare } \\
\text { land }\end{array}$ & 1612 & 1597 & 0.93 & 531 & 67.06 & 1699 & 5.40 \\
\hline $\begin{array}{l}\text { Vege- } \\
\text { table } \\
\text { field }\end{array}$ & 1242 & 1179 & 5.07 & 1980 & 59.42 & 1962 & 57.97 \\
\hline Ditch & 1077 & 1067 & 0.93 & 1695 & 5738 & 1385 & 28.60 \\
\hline $\begin{array}{l}\text { Lotus } \\
\text { root }\end{array}$ & 482 & 427 & 11.41 & 1410 & 192.53 & 567 & 17.63 \\
\hline Trees & 1628 & 1817 & 11.61 & 1157 & 28.93 & 866 & 46.81 \\
\hline $\begin{array}{l}\text { The } \\
\text { way }\end{array}$ & 483 & 561 & 16.15 & 384 & 20.50 & 442 & 8.49 \\
\hline
\end{tabular}
Inception during fine-tuning phases.

\begin{tabular}{|c|c|c|c|c|c|}
\hline \multirow[b]{2}{*}{$\begin{array}{l}\text { Training } \\
\text { Systems }\end{array}$} & \multirow[b]{2}{*}{$\begin{array}{c}\text { Deep Neural } \\
\text { Networks }\end{array}$} & \multicolumn{4}{|c|}{ Performance Indices } \\
\hline & & $\begin{array}{l}\text { Re- } \\
\text { call }\end{array}$ & $\begin{array}{l}\text { Preci- } \\
\text { sion }\end{array}$ & $\begin{array}{l}\text { Accu- } \\
\text { racy }\end{array}$ & $\begin{array}{c}\text { Time } \\
\text { Evaluation } \\
\text { (minutes) }\end{array}$ \\
\hline \multirow{3}{*}{$\begin{array}{c}\text { Fine } \\
\text { Tuning }\end{array}$} & $\begin{array}{c}\text { Inception_V1 } \\
\text { model }\end{array}$ & 100 & 95.35 & 93 & 109 \\
\hline & $\begin{array}{c}\text { Inception_V2 } \\
\text { model }\end{array}$ & 100 & 96.4 & 96.1 & 124 \\
\hline & $\begin{array}{c}\text { Inception_V3 } \\
\text { model }\end{array}$ & 100 & 92.32 & 90.23 & 368 \\
\hline \multirow{3}{*}{$\begin{array}{l}\text { Fully } \\
\text { Training }\end{array}$} & $\begin{array}{c}\text { Inception_V1 } \\
\text { model }\end{array}$ & 100 & 98.37 & 96.32 & 1028 \\
\hline & \begin{tabular}{|c|} 
Inception_V2 \\
model
\end{tabular} & 100 & 100 & 97.24 & 1224 \\
\hline & Inception_V3 & 100 & 100 & 100 & 3365 \\
\hline
\end{tabular}

Table 3: Accuracy of surface area consistency.

Table 4: Performance evaluation of deep neural network for two phase of testing, fine tuning and fully training.

computed for $\mathrm{e}^{\text {th }}$ segment through the deep neural network classifier.

Table 4, presents the statistical parameters that have been utilized for obtaining the average yield of paddy fields. The observations analyzed considering the fact that various bushes of rice are collected manually along $2 \mathrm{~m}^{\wedge} 2$ sample area of paddy field. The number of bushes were counted and analyzed through which the average yield of paddy field is computed.

Figure 5 depicts the training progress considering two different modes, one is fine-tuning and other is full training mode respectively. For a fine-tuning stage, several model of Inception observed converge after reaching the iteration count to 4,000 iterations as depicted in Figure 5. On the other hand for a full training mode, inception models require iteration count of 12,000 for converging. Therefore the training period of these inception models for full training mode is higher in comparison with fine tuning mode.

It is observed from the experimentation that several images are required in order to capture the complete paddy area through UAV's. The altitude of 
the UAV's and configuration of installed IR cameras plays a major role in collecting number of images from UAV's. For the accurate estimation of overall paddy area, process of assessment is evaluated many times corresponding to the amount of images collected, and therefore the overall yield is evaluated as an average of all images.

\section{Discussion}

With the rapid development of "beautiful villages" and "green roads", starting from the protection of farmland and improving the rural living environment, efforts have been made to plant trees, farmland forest nets, and village greening and beautification. By the end of 2017, the national highway greening mileage has reached 2.644 million $\mathrm{km}$, the greening rate reached $63.7 \%$, the traditional GVG collection method along the road will be more and more affected. The portable drone used in this study has a simple take-off and landing method, and has high shooting efficiency. The drone can quickly collect sample image data and overcome the inevitably interference from road trees during the use of the GVG agricultural sampling system. Flying over trees and other obstacles for data collection improves the efficiency of collecting effective photos, and the use of drones for sampling in this article can also obtain data within a certain area for rapid crop area extraction and planting ratio calculation, which is more effective than traditional artificial planting. After the number is judged, the accuracy and efficiency of regional planting ratio calculation are significantly improved. In addition, compared with corn, wheat and other crops, the growing season of rice is rainier, and the optical satellite remote sensing is affected. The resolution of microwave remote sensing images is also low. The timeliness and quality of the data are difficult to guarantee. Inorganic low-altitude remote sensing is more flexible and can overcome this impact. , Supplement some missing data.

Due to the limitations of the battery life and flying height of the portable UAV, the UAV image obtained in this paper is relatively small in width and the range of the sample plot, which makes the topography of the sample plot insignificant and the types of ground features are limited, although there are several visual comparisons. After the segmentation parameter determines the scale optimization range, the ESP tool is used for quantitative analysis, and the segmentation scale is selected as 300 . However, considering the variability of regional terrain, the richness of feature types and the complexity of geographic elements when UAV images are high-resolution, the optimal segmentation scale obtained in this paper is difficult to fit the segmentation scale of various regional features. Therefore, the adaptive multi-scale automatic segmentation algorithm based on the surface characteristics of the UAV image needs to be further developed, so that the segmented image object can achieve a better match with the real ground object, while reducing the need for scale selection time. Subsequent optimization and sorting of the objectoriented classification methods for drone images of different terrains and different crop types can obtain comprehensive, diverse, and high-quality crop type information, and establish training for the use of deep learning for drone image crop classification. The sample data set will improve the automation and intelligence level of image interpretation, promote the expansion of agricultural sampling range and efficiency, and increase the speed of collecting food planting information.

\section{Conclusion}

This paper uses portable UAV to quickly obtain images of rice, lotus root, vegetable field and other crops that are blocked by houses and trees. Using Agisoft PhotoScan software to splice images and generate high-resolution orthophoto images, the best segmentation scale is determined quickly by combining visual judgment and ESP tools, and 6 feature combinations of red spectral mean, yellow spectral standard deviation, blue spectral standard deviation, maximization difference measure, shape density and gray level co-occurrence matrix contrast are selected as the optimal feature space. Objectoriented segmentation and three commonly used supervised classification methods are used to classify objects and extract area quickly. The results and area of nearest neighbor, support vector machine and random forest classification are evaluated by visual interpretation. The highest overall accuracy is the nearest neighbor classification. At this time, the user accuracy of rice classification is $95 \%$ and the accuracy of area consistency is $99.25 \%$.

UAVs can take off and land quickly without being restricted by terrain, and can overcome the shortcomings of the limited number of effective pictures collected by traditional GVG agricultural information collection systems when there are many roadside obstructions, and provide crop planting information for missing areas. Although UAV images only have three kinds of spectral information of red, green and blue, they have clearer shape and texture information than satellite remote sensing images. They are used to calculate the rice planting area of sample plots in plain areas with fast speed and high accuracy, which can be used for large-scale rice cultivation. The calculation of area, yield and other information provides a basis for verification.

\section{References}

[1] Habibi, L. N., Komariah, K., Ariyanto, D. P., Syamsiyah, J., \& Tanaka, T. S. (2019). Estimation of Soil Organic Matter on Paddy Field using Remote Sensing Method. SAINS TANAH-Journal of Soil Science and Agroclimatology, 16(2), 159. 168.

https://doi.org/10.20961/stjssa.v16i2.35395 
[2] Hu, W., Li, C. H., Ye, C., Wang, J., Wei, W. W., \& Deng, Y. (2019). Research progress on ecological models in the field of water eutrophication: CiteSpace analysis based on data from the ISI web of science database. Ecological Modelling, 410, 108779.

https://doi.org/10.1016/j.ecolmodel.2019.108779

[3] Singh, A., \& Kumar, A. (2020). Identification of paddy stubble burnt activities using temporal class-based sensor-independent indices database: modified possibilistic fuzzy classification approach. Journal of the Indian Society of Remote Sensing, 48(3), 423-430. https://doi.org/10.1007/s12524-019-01093-4.

[4] Sharma, A., Singh, P. K., \& Kumar, Y. (2020). An integrated fire detection system using IoT and image processing technique for smart cities. Sustainable Cities and Society, 61, 102332. https://doi.org/10.1016/j.scs.2020.102332

[5] Sharma, A., Singh, P. K., Sharma, A., \& Kumar, R. (2019). An efficient architecture for the accurate detection and monitoring of an event through the sky. Computer Communications, 148, 115-128. https://doi.org/10.1016/j.comcom.2019.09.009

[6] Frolking, S., Qiu, J., Boles, S., Xiao, X., Liu, J., Zhuang, Y., \& Qin, X. (2002). Combining remote sensing and ground census data to develop new maps of the distribution of rice agriculture in China. Global Biogeochemical Cycles, 16(4), 381 . https://doi.org/10.1029/2001GB001425

[7] Liu, H., Zhang, J., Pan, Y., Shuai, G., Zhu, X., \& Zhu, S. (2018). An efficient approach based on UAV orthographic imagery to map paddy with support of field-level canopy height from point cloud data. IEEE Journal of Selected Topics in Applied Earth Observations and Remote Sensing, 11(6), 2034-2046. https://doi.org/10.1109/JSTARS.2018.2829218

[8] Dai, J. G., Zhang, G. S., Guo, P., Zeng, T. J., Cui, M., \& Xue, J. L. (2019). Information extraction of cotton lodging based on multi-spectral image from UAV remote sensing. Trans. CSAE, 35, 63-70. https://doi.org/10.1016/j.scs.2020.102332

[9] Zhang, F., Jing, Z. X., Ji, B. Y., Pei, L. X., Chen, S. Q., Wang, X. Y., \& Huang, L. Q. (2019). Study of extracting natural resources of Chinese medicinal materials planted area in Luoning of Henan province based on UAV of low altitude remote sensing technology and remote sensing image of satellite. Zhongguo Zhong yao za zhi= Zhongguo zhongyao zazhi= China journal of Chinese materia medica, 44(19), 4095-4100. https://doi.org/10.1016/j.scs.2020.102332

[10] Shafi, U., Mumtaz, R., García-Nieto, J., Hassan, S. A., Zaidi, S. A. R., \& Iqbal, N. (2019). Precision agriculture techniques and practices: From considerations to applications. Sensors, 19(17), 3796.

https://doi.org/10.3390/s19173796
[11] Mekonnen, Y., Namuduri, S., Burton, L., Sarwat, A., \& Bhansali, S. (2019). Machine learning techniques in wireless sensor network based precision agriculture. Journal of the Electrochemical Society, 167(3), 037522. https://doi.org/10.1149/2.0222003JES

[12] Dhillon, S., Madhu, C., Kaur, D., \& Singh, S. (2020). A solar energy forecast model using neural networks: Application for prediction of power for wireless sensor networks in precision agriculture. Wireless Personal Communications, 1-20. https://doi.org/10.1007/s11277-020-07173-w

[13] Mazloumzadeh, S. M., Shamsi, M., \& Nezamabadi-Pour, H. (2010). Fuzzy logic to classify date palm trees based on some physical properties related to precision agriculture. Precision agriculture, 11(3), 258-273. https://doi.org/10.1007/s11119-009-9132-2

[14] Pudumalar, S., Ramanujam, E., Rajashree, R. H., Kavya, C., Kiruthika, T., \& Nisha, J. (2017, January). Crop recommendation system for precision agriculture. In 2016 Eighth International Conference on Advanced Computing (ICoAC) (pp. 32-36). IEEE. https://doi.org/10.1109/ICoAC.2017.7951740

[15] Guerrero, J. M., Pajares, G., Montalvo, M., Romeo, J., \& Guijarro, M. (2012). Support vector machines for crop/weeds identification in maize fields. Expert Systems with Applications, 39(12), 11149-11155. https://doi.org/10.1016/j.eswa.2012.03.040

[16] Abdullahi, H. S., Sheriff, R., \& Mahieddine, F. (2017, August). Convolution neural network in precision agriculture for plant image recognition and classification. In 2017 Seventh International Conference on Innovative Computing Technology (INTECH) (Vol. 10). Ieee. https://doi.org/10.1109/INTECH.2017.8102436

[17] Candiago, S., Remondino, F., De Giglio, M., Dubbini, M., \& Gattelli, M. (2015). Evaluating multispectral images and vegetation indices for precision farming applications from UAV images. Remote sensing, 7(4), 4026-4047. https://doi.org/10.3390/rs70404026

[18] Khanal, S., Fulton, J., \& Shearer, S. (2017). An overview of current and potential applications of thermal remote sensing in precision agriculture. Computers and Electronics in Agriculture, 139, 22-32.

https://doi.org/10.1016/j.compag.2017.05.001

[19] Mahlein, A. K. (2016). Plant disease detection by imaging sensors-parallels and specific demands for precision agriculture and plant phenotyping. Plant disease, 100(2), 241-251. https://doi.org/10.1094/PDIS-03-15-0340-FE

[20] Panda, S. S., Ames, D. P., \& Panigrahi, S. (2010). Application of vegetation indices for agricultural crop yield prediction using neural network techniques. Remote Sensing, 2(3), 673-696. https://doi.org/10.3390/rs2030673 
[21] Becker-Reshef, I., Vermote, E., Lindeman, M., \& Justice, C. (2010). A generalized regression-based model for forecasting winter wheat yields in Kansas and Ukraine using MODIS data. Remote sensing of environment, 114(6), 1312-1323. https://doi.org/10.1016/j.rse.2010.01.010

[22] Kolesau, A., \& Šešok, D. (2020). Voice activation systems for embedded devices: Systematic literature review. Informatica, 31(1), 65-88, DOI: https://doi.org/10.15388/20-INFOR398.

[23] Sharma, A., Tomar, R., Chilamkurti, N., \& Kim, B. G. (2020). Blockchain based smart contracts for internet of medical things in e-healthcare. Electronics, 9(10), 1609.

https://doi.org/10.3390/electronics9101609

Poongodi, M., Sharma, A., Vijayakumar, V., Bhardwaj, V., Sharma, A. P., Iqbal, R., \& Kumar, R. (2020). Prediction of the price of Ethereum blockchain cryptocurrency in an industrial finance system. Computers \& Electrical Engineering, 81, 106527. https://doi.org/10.1016/j.compeleceng. 2019.106527

[24] Vaitkevicius, P., \& Marcinkevicius, V. (2020). Comparison of classification algorithms for detection of phishing websites. Informatica, 31(1), 143-160, DOI: https://doi.org/10.15388/20-INFOR404

[25] Cao, Y., Li, G. L., Luo, Y. K., Pan, Q., \& Zhang, S. Y. (2020). Monitoring of sugar beet growth indicators using wide-dynamic-range vegetation index (WDRVI) derived from UAV multispectral images. Computers and Electronics in Agriculture, 171, 105331. https://doi.org/10.1016/j.compag.2020.105331

[26] Shrestha, R., Di, L., Eugene, G. Y., Kang, L., SHAO, Y. Z., \& BAI, Y. Q. (2017). Regression model to estimate flood impact on corn yield using MODIS NDVI and USDA cropland data layer. Journal of Integrative Agriculture, 16(2), 398-407. https://doi.org/10.1016/S2095-3119(16)61502-2.

[27] Noje, D., Dzitac, I., Pop, N., \& Tarca, R. (2020). IoT devices signals processing based on Shepard local approximation operators defined in Riesz MV-algebras. Informatica, 31(1), 131-142, DOI: https://doi.org/10.15388/20-INFOR395.

[28] Sharma, A., \& Singh, P. K. (2020). Taxonomy on localization issues and challenges in wireless sensor networks. Recent Advances in Electrical \& Electronic Engineering (Formerly Recent Patents on Electrical \& Electronic Engineering), 13(2), 193-202. https://doi.org/10.2174/ 2352096512666190212153057

[29] Sharma, A., \& Singh, P. K. (2021). Localization in Wireless Sensor Networks for Accurate Event Detection. International Journal of Healthcare Information Systems and Informatics (IJHISI), 16(3), 74-88. https://doi.org/10.4018/IJHISI.20210701.oa5

[30] Deng, L., Mao, Z., Li, X., Hu, Z., Duan, F., \& Yan, Y. (2018). UAV-based multispectral remote sensing for precision agriculture: A comparison between different cameras. ISPRS journal of photogrammetry and remote sensing, 146, 124136.

https://doi.org/10.1016/j.isprsjprs.2018.09.008

[31] Al-Ykoob, S. M., \& Sherali, H. D. (2020). A Complementary Column Generation Approach for the Graph Equipartition Problem. Informatica, 31(1), 1-20,

DOI: https://doi.org/10.15388/20-INFOR391.

[32] Rokhmana, C. A. (2015). The potential of UAVbased remote sensing for supporting precision agriculture in Indonesia. Procedia Environmental Sciences, 24, 245-253. https://doi.org/10.1016/j.proenv.2015.03.032

[33] Kim, J., Ryu, Y., Jiang, C., \& Hwang, Y. (2019). Continuous observation of vegetation canopy dynamics using an integrated low-cost, nearsurface remote sensing system. Agricultural and forest meteorology, 264, 164-177. https://doi.org/10.1016/j.agrformet.2018.09.014

[34]Fang, H., Chen, H., Jiang, H., Wang, Y., Liu, Y., Liu, F., \& He, Y. (2019). Research on Method of Farmland Obstacle Boundary Extraction in UAV Remote Sensing Images. Sensors, 19(20), 4431. https://doi.org/10.3390/s19204431

[35] Wang, Y., Zhang, K., Tang, C., Cao, Q., Tian, Y., Zhu, Y., \& Liu, X. (2019). Estimation of rice growth parameters based on linear mixed-effect model using multispectral images from fixed-wing unmanned aerial vehicles. Remote sensing, 11(11), 1371. https://doi.org/10.3390/rs11111371. 
